\title{
Sequence analysis of the catalytic subunit of PKA in somatotroph adenomas
}

\author{
Sarah J Larkin, Francesco Ferraù ${ }^{1}$, Niki Karavitaki ${ }^{2}$, Laura C Hernández-Ramírez ${ }^{1}$, \\ Olaf Ansorge $^{\dagger}$, Ashley B Grossman ${ }^{2,+}$ and Márta Korbonits ${ }^{1,+}$ \\ Nuffield Department of Clinical Neurosciences, Department of Neuropathology, Level 1 West Wing, John Radcliffe \\ Hospital, Headley Way, Oxford OX3 9DU, UK, 'Department of Endocrinology, Barts and London School of Medicine, \\ Queen Mary University of London, London EC1A 6BQ, UK and ${ }^{2}$ Department of Endocrinology, Oxford Centre for \\ Diabetes, Endocrinology and Metabolism, Churchill Hospital, Old Road, Headington, Oxford OX3 7LE, UK \\ ${ }^{\dagger}$ (O Ansorge, A B Grossman and M Korbonits considered as joint senior authors)
}

\author{
Correspondence \\ should be addressed \\ to S J Larkin \\ Email \\ sarah.larkin@ndcn.ox.ac.uk
}

\begin{abstract}
Objective: The pathogenetic mechanisms of sporadic somatotroph adenomas are not well understood, but derangements of the CAMP pathway have been implicated. Recent studies have identified L206R mutations in the alpha catalytic subunit of protein kinase $\mathrm{A}$ (PRKACA) in cortisol-producing adrenocortical adenomas and amplification of the beta catalytic subunit of protein kinase $A P R K A C B$ in acromegaly associated with Carney complex. Given that both adrenocortical adenomas and somatotroph adenomas are known to be reliant on the CAMP signalling pathway, we sought to determine the relevance of the L206R mutation in both PRKACA and PRKACB for the pathogenesis of sporadic somatotroph adenomas.

Design: Somatotroph adenoma specimens, both frozen and formalin-fixed, from patients who underwent surgery for their acromegaly between 1995 and 2012, were used in the study.

Methods: The DNA sequence at codon 206 of PRKACA and PRKACB was determined by PCR amplification and sequencing. The results were compared with patient characteristics, the mutational status of the GNAS complex locus and the tumour granulation pattern.

Results: No mutations at codon 206 of PRKACA or PRKACB were found in a total of 92 specimens, comprising both WT and mutant GNAS cases, and densely, sparsely and mixed granulation patterns.

Conclusions: It is unlikely that mutation at this locus is involved in the pathogenesis of sporadic somatotroph adenoma; however, gene amplification or mutations at other loci or in other components of the cAMP signalling pathway, while unlikely, cannot be ruled out.
\end{abstract}

\section{Introduction}

Genetic studies of the pathogenesis of sporadic somatotroph adenomas have identified a role for the cAMP signalling pathway, and conversely syndromes that feature a germline mutation in the components of this pathway can be associated with the formation of somatotroph adenomas. Thus, sporadic somatotroph adenomas are often associated with mutations at the GNAS complex locus, encoding the GSP oncogene. These mutations implicate the cAMP pathway in the pathogenesis of sporadic somatotroph adenomas in 15-58\% of cases $(1,2,3)$, although the tumourigenic mechanism is unclear. The McCune-Albright syndrome results from post-zygotic germline mutations in GNAS and is often characterised by endocrine neoplasia: it is associated with somatotroph adenomas and acromegaly in 10-20\% of cases (4). Carney complex is an autosomal dominant syndrome characterised by multiple, often endocrine tumours, including somatotroph adenomas, in $\sim 10 \%$ of cases (5). In over $60 \%$ of cases of Carney complex, a mutation is found in the regulatory subunit $1 \mathrm{~A}$ of protein
(C) 2014 European Society of Endocrinology Printed in Great Britain
Published by Bioscientifica Ltd. 
kinase A (PRKAR1A), an intrinsic part of cAMP signalling, although such mutations are not found in sporadic pituitary adenomas $(6,7,8)$. Recently, whole-exome sequencing studies have identified a mutation in the active-site cleft of catalytic subunit A of PKA (PRKACA) in cortisol-producing adrenocortical adenomas, most commonly R206 $(9,10,11)$. This mutation was observed in $37-66 \%$ of cases and resulted in constitutive activation of PKA that was neither suppressed by the PKA regulatory subunit nor increased by addition of cAMP (either directly or indirectly by forskolin treatment) $(10,11)$. A recent report of a patient with Carney complex with acromegaly, spotty pigmentation and myxomas, but without Cushing's syndrome, has shown the presence of a triplication of chromosome 1p31.1 (containing PRKACB), leading to increased expression of $P R K A C B$ (12). The authors propose that amplification of $P R K A C B$ may be responsible for non-adrenal manifestations of Carney complex. Given the involvement of the cAMP pathway in sporadic somatotroph adenoma pathogenesis, we sought to determine whether mutations in the catalytic subunit of PKA might also occur in these lesions and be involved in the pathogenesis of these tumours. We therefore amplified and sequenced the region of PRKACA and PRKACB containing codon 206 in a large series of sporadic somatotroph adenomas. The findings were compared with patient characteristics, the mutational status of GNAS in a sub-group and the granulation pattern of the specimens.

\section{Subjects and methods}

Patients $(n=92)$ with a diagnosis of acromegaly were included. None of the patients had a clinical history of FIPA, MEN1 or SDH-associated acromegaly, but germline mutations in MEN1 and SDH were not sought routinely in all individuals. The tumour samples, either formalin fixed and paraffin embedded (FFPE; $n=43)$ or frozen $(n=49)$, were retrieved from the surgical neuropathology archive of the Oxford Brain Bank and the Tissue Bank of the Department of Endocrinology at Barts and the London School of Medicine. The specimens were excluded if the samples were too small or the DNA was of insufficient quality to enable successful amplification of the regions containing codon 206 of PRKACA and PRKACB. The specimens were preferentially included if previous analysis had shown them to be GNAS WT. The findings of Sato et al. (11) demonstrate that mutations in PRKACA and GNAS are mutually exclusive in adrenocortical adenomas and so we speculated that any PRKACA mutations identified might only be present in GNAS-mutation negative specimens. All studies were conducted on linked-anonymised samples under multi-site and local Research Ethics Committee (REC) approval.

DNA was extracted from either $5 \times 10 \mu \mathrm{m}$ sections of FFPE tissue (QiaAmp FFPE DNA Kit, Qiagen) or from homogenised fresh-frozen specimens (QiaAmp DNA Mini Kit or DNeasy Blood and Tissue Kit (Qiagen)) according to manufacturer's instructions. PCR was carried out to generate amplicons including codon 206 of PRKACA and $P R K A C B$. The primers were designed using Primer-Blast (www.ncbi.nlm.nih.gov/tools/primer-blast). For FFPE specimens primers were as follows: PRKACA sense (5'-GGTGACAGACTTCGGTTTCGC-3') and antisense $\left(5^{\prime}\right.$-CCTTGTTGTAGCCCTGGAGCA-3'); PRKACB sense (5'-TGGTTTTATTTCTTTGCAGTGAGC-3') and antisense (5'-CCTGGATATAGCCTTGATGGTCA-3'). The primers for gDNA from frozen specimens were as follows: PRKACA sense (5'-CAACTGCCTGTTCTTGTGCC-3 $\left.{ }^{\prime}\right)$ and antisense (5'-AGTCCACGGCCTTGTTGTAG-3'); PRKACB sense $\left(5^{\prime}\right.$-AAACTTTCAACGTAGGTGCAAT-3 $\left.{ }^{\prime}\right)$ and antisense $\left(5^{\prime}\right.$-CAAAAGTCCATAGGGATGCATGT- $\left.3^{\prime}\right)$. The primers for cDNA from frozen specimens were as follows: PRKACA sense (5'-CTGCACTCGCTGGATCTCAT-3') and antisense (5'-CAGAGCTGAAGTGGGAAGGG-3') and PRKACB sense (5'-GCAGCTCAGATAGTGCTAACATTC- $\left.3^{\prime}\right)$ and antisense (5'-GGTCTGCAAAGAATGGGGGATA-3').

For FFPE specimens, DNA template (100 ng) was added to $10 \times$ PCR buffer solution (10\% v/v; Qiagen), $\mathrm{MgCl}_{2}$ (final concentration $4 \mathrm{mmol} / \mathrm{l}$, for $P R K A C B$ only), dNTPs $(200 \mu \mathrm{mol} / \mathrm{l})$ (Promega), $0.5 \mathrm{U}$ of Taq polymerase (HotStarTaq Plus, Qiagen) and $400 \mathrm{nmol} / \mathrm{l}$ each of forward and reverse primers. The cycling conditions were as follows: $95^{\circ} \mathrm{C}$ for $5 \mathrm{~min}$, followed by 35 cycles of $95^{\circ} \mathrm{C}$ for $30 \mathrm{~s}$; annealing temperature for $30 \mathrm{~s}\left(61^{\circ} \mathrm{C}\right.$ for PRKACA and $57^{\circ} \mathrm{C}$ for $P R K A C B$ ) and $72{ }^{\circ} \mathrm{C}$ for $40 \mathrm{~s}$, followed by a single 1 min extension. Total reaction volume was $20 \mu \mathrm{l}$. For frozen specimens, gDNA template ((100 ng) was added to $10 \times$ Taq reaction buffer $(10 \% \mathrm{v} / \mathrm{v}$; (New England Biolabs (NEB), Hitchin, UK)), dNTPs $(200 \mu \mathrm{mol} / \mathrm{l})$ (New England Biolabs), $0.12 \mathrm{U}$ of Taq polymerase (New England Biolabs) and $200 \mathrm{nmol} / \mathrm{l}$ each of forward and reverse primers. The cycling conditions were as follows: $95^{\circ} \mathrm{C}$ for $2 \mathrm{~min}$, followed by 35 cycles at $95^{\circ} \mathrm{C}$ for $30 \mathrm{~s}$, annealing temperature for $30 \mathrm{~s}\left(67.6^{\circ} \mathrm{C}\right.$ for $P R K A C A$ and $62.5^{\circ} \mathrm{C}$ for $P R K A C B$ ) and $68^{\circ} \mathrm{C}$ for $30 \mathrm{~s}$, followed by a single $5 \mathrm{~min}$ extension. Total reaction volume was $25 \mu \mathrm{l}$.

RNA was extracted from frozen specimens using the RNeasy Kit (Qiagen), according to manufacturer's instructions. RT was performed using $500 \mathrm{ng}$ RNA, random hexamer primers $(0.5 \mu \mathrm{g} / \mathrm{l}$, Promega) and M-MLV reverse 
Table 1 Cases included in the study. Cases 1-21 have previously been reported (13).

\begin{tabular}{|c|c|c|c|c|c|c|c|c|c|}
\hline Case & Sex & Age & Material & Preservation & Size & Granulation & GNAS & PRKACA & PRKACB \\
\hline 1 & $\mathrm{~F}$ & 39 & gDNA & FFPE & Macro & $\mathrm{S}$ & WT & WT & WT \\
\hline 2 & $\mathrm{~F}$ & 64 & gDNA & FFPE & Micro & D & WT & WT & WT \\
\hline 3 & $\mathrm{~F}$ & 54 & gDNA & FFPE & Macro & $\mathrm{D}$ & WT & WT & WT \\
\hline 4 & $\mathrm{~F}$ & 41 & gDNA & FFPE & Micro & $\mathrm{D}$ & WT & WT & WT \\
\hline 5 & $\mathrm{~F}$ & 57 & gDNA & FFPE & Macro & D & WT & WT & WT \\
\hline 6 & $M$ & 49 & gDNA & FFPE & Macro & $\mathrm{D}$ & WT & WT & WT \\
\hline 7 & $\mathrm{~F}$ & 56 & gDNA & FFPE & Micro & $\mathrm{M}$ & WT & WT & WT \\
\hline 8 & $M$ & 35 & gDNA & FFPE & Macro & ND & R201C & WT & WT \\
\hline 9 & $\mathrm{~F}$ & 66 & gDNA & FFPE & Macro & $S$ & ND & WT & WT \\
\hline 10 & $\mathrm{~F}$ & 23 & gDNA & FFPE & Macro & $S$ & ND & WT & WT \\
\hline 11 & $\mathrm{~F}$ & 47 & gDNA & FFPE & Macro & $\mathrm{S}$ & WT & WT & WT \\
\hline 12 & $M$ & 43 & gDNA & FFPE & Micro & D & WT & WT & WT \\
\hline 13 & $\mathrm{~F}$ & 44 & gDNA & FFPE & Macro & $S$ & WT & WT & WT \\
\hline 14 & $\mathrm{~F}$ & 62 & gDNA & FFPE & Macro & $M$ & WT & WT & WT \\
\hline 15 & $M$ & 52 & gDNA & FFPE & Macro & $S$ & $\mathrm{R} 102 \mathrm{C}$ & WT & WT \\
\hline 16 & $\mathrm{~F}$ & 54 & gDNA & FFPE & Macro & $M$ & WT & WT & WT \\
\hline 17 & $\mathrm{~F}$ & 26 & gDNA & FFPE & Macro & $S$ & ND & WT & WT \\
\hline 18 & $\mathrm{~F}$ & 33 & gDNA & FFPE & Macro & ND & WT & WT & WT \\
\hline 19 & $\mathrm{~F}$ & 53 & gDNA & FFPE & Macro & ND & WT & WT & WT \\
\hline 20 & $\mathrm{~F}$ & 59 & gDNA & FFPE & Micro & $\mathrm{D}$ & WT & WT & WT \\
\hline 21 & $M$ & 56 & gDNA & Frozen & Macro & D & R201C & WT & WT \\
\hline 22 & $\mathrm{~F}$ & 49 & gDNA & FFPE & ND & $\mathrm{S}$ & ND & WT & WT \\
\hline 23 & $M$ & 31 & gDNA & FFPE & ND & $S$ & ND & WT & WT \\
\hline 24 & $\mathrm{~F}$ & 45 & gDNA & FFPE & ND & ND & ND & WT & WT \\
\hline 25 & $\mathrm{~F}$ & 49 & gDNA & FFPE & ND & ND & ND & WT & WT \\
\hline 26 & $M$ & 34 & gDNA & FFPE & Micro & $S$ & ND & WT & WT \\
\hline 27 & $M$ & 55 & gDNA & FFPE & Micro & $S$ & ND & WT & WT \\
\hline 28 & $\mathrm{M}$ & 43 & gDNA & FFPE & Micro & ND & ND & WT & WT \\
\hline 29 & $\mathrm{~F}$ & 46 & gDNA & FFPE & Macro & ND & ND & WT & WT \\
\hline 30 & $\mathrm{~F}$ & 61 & gDNA & FFPE & Macro & $S$ & ND & WT & WT \\
\hline 31 & $\mathrm{~F}$ & 19 & gDNA & FFPE & Macro & ND & ND & WT & WT \\
\hline 32 & $\mathrm{~F}$ & 41 & gDNA & FFPE & Macro & $D$ & ND & WT & WT \\
\hline 33 & $M$ & 52 & gDNA & FFPE & ND & ND & ND & WT & WT \\
\hline 34 & $\mathrm{~F}$ & 29 & gDNA & FFPE & Micro & ND & ND & WT & WT \\
\hline 35 & $M$ & 40 & gDNA & FFPE & ND & ND & ND & WT & WT \\
\hline 36 & $M$ & 69 & gDNA & FFPE & ND & ND & ND & WT & WT \\
\hline 37 & $M$ & 18 & gDNA & FFPE & Macro & ND & ND & WT & WT \\
\hline 38 & $\mathrm{~F}$ & 41 & gDNA & FFPE & Micro & $D$ & ND & WT & WT \\
\hline 39 & $\mathrm{~F}$ & 25 & gDNA & FFPE & Macro & $\mathrm{S}$ & ND & WT & WT \\
\hline 40 & $\mathrm{~F}$ & 29 & gDNA & FFPE & Macro & $\mathrm{S}$ & ND & WT & WT \\
\hline 41 & $\mathrm{~F}$ & 67 & gDNA & FFPE & Macro & $\mathrm{D}$ & ND & WT & WT \\
\hline 42 & $M$ & 53 & gDNA & FFPE & Macro & ND & WT & WT & WT \\
\hline 43 & $\mathrm{~F}$ & 30 & gDNA & FFPE & Macro & $S$ & WT & WT & WT \\
\hline 44 & $\mathrm{~F}$ & 36 & gDNA & FFPE & Macro & $\mathrm{S}$ & WT & WT & WT \\
\hline 45 & $\mathrm{~F}$ & 57 & gDNA & Frozen & Macro & ND & ND & WT & WT \\
\hline 46 & $M$ & 48 & gDNA & Frozen & Micro & ND & ND & WT & WT \\
\hline 47 & $\mathrm{~F}$ & 42 & gDNA & Frozen & Macro & ND & ND & WT & WT \\
\hline 48 & $M$ & 50 & gDNA & Frozen & Micro & ND & WT & WT & WT \\
\hline 49 & $M$ & 80 & gDNA & Frozen & Macro & ND & ND & WT & WT \\
\hline 50 & $\mathrm{~F}$ & 66 & gDNA & Frozen & Macro & ND & ND & WT & WT \\
\hline 51 & $\mathrm{~F}$ & 37 & gDNA & Frozen & Macro & ND & ND & WT & WT \\
\hline 52 & $M$ & 32 & gDNA & Frozen & Macro & ND & ND & WT & WT \\
\hline 53 & $M$ & 71 & gDNA & Frozen & Macro & ND & ND & WT & WT \\
\hline 54 & $\mathrm{~F}$ & 53 & gDNA & Frozen & ND & ND & ND & WT & WT \\
\hline 55 & $M$ & 42 & gDNA & Frozen & Macro & ND & ND & WT & WT \\
\hline 56 & $\mathrm{~F}$ & 33 & gDNA & Frozen & Macro & ND & ND & WT & WT \\
\hline 57 & $M$ & 44 & gDNA & Frozen & Macro & ND & ND & WT & WT \\
\hline 58 & $\mathrm{~F}$ & 38 & gDNA & Frozen & Macro & ND & ND & WT & WT \\
\hline 59 & $M$ & 48 & gDNA & Frozen & ND & ND & ND & WT & WT \\
\hline 60 & $\mathrm{~F}$ & 28 & gDNA & Frozen & Macro & ND & ND & WT & WT \\
\hline 61 & $M$ & 40 & gDNA & Frozen & ND & ND & ND & WT & WT \\
\hline
\end{tabular}


Table 1 Continued

\begin{tabular}{|c|c|c|c|c|c|c|c|c|c|}
\hline Case & Sex & Age & Material & Preservation & Size & Granulation & GNAS & PRKACA & PRKACB \\
\hline 62 & $\mathrm{~F}$ & 33 & gDNA & Frozen & Micro & ND & ND & WT & WT \\
\hline 63 & $\mathrm{~F}$ & 43 & gDNA & Frozen & Macro & ND & ND & WT & WT \\
\hline 64 & $\mathrm{~F}$ & 26 & gDNA & Frozen & Micro & ND & ND & WT & WT \\
\hline 65 & $\mathrm{M}$ & 63 & gDNA & Frozen & Macro & ND & ND & WT & WT \\
\hline 66 & $F$ & 57 & gDNA & Frozen & Macro & ND & ND & WT & WT \\
\hline 67 & $\mathrm{M}$ & 48 & gDNA & Frozen & Micro & ND & ND & WT & WT \\
\hline 68 & $\mathrm{~F}$ & 46 & gDNA & Frozen & ND & ND & ND & WT & WT \\
\hline 69 & $\mathrm{~F}$ & 32 & gDNA & Frozen & Macro & ND & ND & WT & WT \\
\hline 70 & $\mathrm{~F}$ & 25 & gDNA & Frozen & Macro & ND & ND & WT & WT \\
\hline 71 & $\mathrm{M}$ & 25 & gDNA & Frozen & Macro & ND & ND & WT & WT \\
\hline 72 & $\mathrm{M}$ & 10 & gDNA & Frozen & Macro & ND & ND & WT & WT \\
\hline 73 & $\mathrm{~F}$ & 14 & gDNA & Frozen & Macro & ND & ND & WT & WT \\
\hline 74 & $M$ & 62 & gDNA & Frozen & Macro & ND & ND & WT & WT \\
\hline 75 & $\mathrm{~F}$ & 48 & gDNA & Frozen & Micro & ND & ND & WT & WT \\
\hline 76 & $\mathrm{~F}$ & 63 & gDNA & Frozen & Macro & ND & ND & WT & WT \\
\hline 77 & $F$ & 57 & gDNA & Frozen & ND & ND & ND & WT & WT \\
\hline 78 & M & 34 & gDNA & Frozen & Macro & ND & ND & WT & WT \\
\hline 79 & M & 26 & gDNA & Frozen & Macro & ND & ND & WT & WT \\
\hline 80 & $\mathrm{~F}$ & 28 & gDNA & Frozen & ND & ND & ND & WT & WT \\
\hline 81 & $\mathrm{~F}$ & 40 & gDNA & Frozen & Macro & ND & ND & WT & WT \\
\hline 82 & $\mathrm{~F}$ & 78 & gDNA & Frozen & Micro & ND & ND & WT & WT \\
\hline 83 & M & 39 & gDNA & Frozen & Macro & ND & ND & WT & WT \\
\hline 84 & M & 53 & gDNA & Frozen & Macro & ND & ND & WT & WT \\
\hline 85 & $M$ & 53 & cDNA & Frozen & Macro & ND & WT & WT & WT \\
\hline 86 & M & ND & CDNA & Frozen & Macro & ND & WT & WT & WT \\
\hline 87 & M & 69 & cDNA & Frozen & Macro & ND & WT & WT & WT \\
\hline 88 & M & 62 & CDNA & Frozen & Micro & ND & R201C & WT & WT \\
\hline 89 & $M$ & 40 & cDNA & Frozen & Macro & ND & ND & WT & WT \\
\hline 90 & $M$ & 52 & cDNA & Frozen & Macro & ND & ND & WT & WT \\
\hline 91 & $\mathrm{~F}$ & 41 & cDNA & Frozen & Macro & ND & ND & WT & WT \\
\hline 92 & $\mathrm{~F}$ & 64 & CDNA & Frozen & Macro & ND & ND & WT & WT \\
\hline
\end{tabular}

ND, not determined; DG, densely granulated; MG, mixed granulation pattern; SG, sparsely granulated; FFPE, formalin fixed, paraffin embedded; macro macroadenoma; micro, microadenoma.

transcriptase (100 U) (Life Technologies). For amplification of PRKACA, cDNA template (100 ng) was added to $5 \times$ Q5 reaction buffer (20\% v/v; (New England Biolabs)), $0.25 \mu 1$ DNA polymerase (Q5 High-Fidelity DNA polymerase, New England Biolabs) $5 \times$ Q5 High GC Enhancer (20\% v/v, New England Biolabs) dNTPs $(200 \mu \mathrm{mol} / \mathrm{l})$ and $500 \mathrm{nmol} / \mathrm{l}$ each of forward and reverse primers. The cycling conditions were as follows: $98^{\circ} \mathrm{C}, 30 \mathrm{~s}$, followed by 35 cycles of $98^{\circ} \mathrm{C}$ for $10 \mathrm{~s}, 60^{\circ} \mathrm{C}$ for $20 \mathrm{~s}, 72{ }^{\circ} \mathrm{C}$ for $20 \mathrm{~s}$ followed by a single extension for $2 \mathrm{~min}$. Total reaction volume was $25 \mu \mathrm{l}$. For amplification of $P R K A C B$, reaction and cycling conditions were identical to those for gDNA except that $400 \mu \mathrm{mol} / 1 \mathrm{dNTPs}$ were added and the annealing temperature was $62^{\circ} \mathrm{C}$.

The products were examined by agarose gel separation and purified (MinElute PCR Purification Kit (Qiagen)). Bidirectional sequencing of amplicons was carried out using BigDye Terminator chemistry (v3.1) and an ABI-3730 capillary sequencer (Applied Biosystems). The sequences were compared with the WT sequence (RefSeq NM_002730.3 (PRKACA) and NM_182948.2 (PRKACB)).

The tumours were categorised as sparsely granulated (SG), densely granulated (DG) or of mixed granulation pattern (MG). A mixed phenotype was assigned when more than $30 \%$ of tumour cells deviated from the dominant Cam5.2 pattern. The immunohistochemical methods for determination of granulation pattern and the sequencing of GNAS have been previously described (13).

\section{Results}

In this study, a total of 92 sporadic somatotroph adenoma specimens ( 54 from females) were used. Age at diagnosis varied between 10 and 80 years, and $77 \%$ of samples were obtained from patients with macroadenomas (where tumour size was known; $n=77$ ). There were mutations at the GNAS complex locus, G-protein $\alpha$ subunit codon 201 (R201C), in four of 26 cases for which sequences 
were available (20 from FFPE specimens and six from frozen specimens) (Table 1 ). In the remaining FFPE specimens $(n=23)$, DNA quality was not sufficient to allow amplification of codons 201 and 227 of G-protein $\alpha$ subunit, so such data for these cases were not available.

We did not find a mutation at codon 206 of PRKACA or $P R K A C B$ in either the gDNA or cDNA of any of the sporadic somatotroph adenomas. Although information regarding the mutational status of GNAS and the granulation pattern was not available for all cases, we did not find a mutation in either GNAS mutant or WT cases (mutational status was established in 26 of 92 specimens) or in tumours with any type of granulation pattern.

\section{Discussion}

The lack of a mutation in either PRKACA or $P R K A C B$ in our series suggests that constitutive activation of $P R K A C A$ or $P R K A C B$ by mutation of codon 206 is unlikely to be involved in the pathogenesis of sporadic somatotroph adenomas. However, as we did not sequence all exons of PRKACA or PRKACB, we cannot rule out mutations at other loci, although this seems unlikely.

Both adrenal adenomas and pituitary somatotroph adenomas are known to contain mutations in components of the cAMP signalling pathway in a proportion of cases, and mutations in PRKAR1A can underlie the development of both of these lesions. However, while mutation of PRKACA appears to be important for the development of up to two-thirds of cortisol-producing adrenal adenomas $(9,10)$, it does not appear to be the case for somatotroph adenomas, even though mutation of the cAMP signalling pathway has been shown to be involved in the pathogenesis of this tumour type $(1,2,13,14,15$, $16,17,18,19)$. We did not determine whether there was amplification of either the PRKACA or PRKACB gene in this series. Amplification of chromosome 19p (containing PRKACA) and 1p31.1 has been shown to underlie impaired inhibition of the catalytic subunit by the PKA regulatory subunit and increased basal PKA activity $(9,12)$. In the absence of a PRKACA or PRKACB mutation, amplification could lead to increased cAMP signalling that might result in somatotroph tumourigenesis.

In adrenocortical adenomas, mutations in PRKACA have been found to be mutually exclusive with mutations in GNAS (11). In this series, we found no mutation in codon 206 of PRKACA or PRKACB in 22 specimens which were GNAS WT and four specimens that had GNAS R201C mutations. Where GNAS status was known, we preferentially included WT specimens in this series. However, there is a lack of information concerning the mutational status of GNAS in the remaining cases $(n=66)$. Given the reported prevalence of GNAS mutations in somatotroph adenomas $(1,2,3)$, we could expect $15-58 \%$ of them (between ten and 38 specimens) to harbour a GNAS mutation; this suggests that although the majority would be negative for GNAS mutation, GNAS mutation in a subset may preclude the finding of a PRKACA or PRKACB mutation.

Previously, we and others have suggested that DG and SG somatotroph adenomas may have differing clinical characteristics and responses to treatment $(1,18,20$, $21,22)$. The genetic basis for this difference has yet to be determined, and so a series containing representative samples from all granulation subtypes was chosen for this study.

The findings of several groups $(9,10,11,12)$ support a central role for the cAMP signalling pathway in the pathogenesis of adrenocortical adenomas and somatotroph adenomas. Although we did not demonstrate the presence of a recurring mutation in either PRKACA or PRKACB, mutations in other components of this pathway cannot be ruled out and warrant further investigation. At present, any mutational event pathogenetic in the majority of sporadic somatotroph tumours remains elusive.

\section{Declaration of interest}

The authors declare that there is no conflict of interest that could be perceived as prejudicing the impartiality of the research reported.

\section{Funding}

The research was funded by the National Institute for Health Research (NIHR) Oxford Biomedical Research Centre based at Oxford University Hospitals NHS Trust and University of Oxford and by NIHR and grant support from Pfizer to M Korbonits' laboratory. L C Hernández-Ramírez is supported by the National Council of Science and Technology of Mexico and by the Barts and The London Charity. The views expressed are those of the author(s) and not necessarily those of the NHS, the NIHR or the Department of Health.

\section{Acknowledgements}

The authors acknowledge the Oxford Brain Bank, supported by the Medical Research Council (MRC), Brains for Dementia Research (BDR) and the NIHR Oxford Biomedical Research Centre.

\section{References}

1 Bakhtiar Y, Hirano H, Arita K, Yunoue S, Fujio S, Tominaga A, Sakoguchi T, Sugiyama K, Kurisu K, Yasufuku-Takano J et al. 
Relationship between cytokeratin staining patterns and clinicopathological features in somatotropinomae. European Journal of Endocrinology 2010163 531-539. (doi:10.1530/EJE-10-0586)

2 Landis CA, Masters SB, Spada A, Pace AM, Bourne HR \& Vallar L. GTPase inhibiting mutations activate the alpha chain of Gs and stimulate adenylyl cyclase in human pituitary tumours. Nature 1989 340 692-696. (doi:10.1038/340692a0)

3 Taboada GF, Luque RM, Neto LV, Machado Ede O, Sbaffi BC, Domingues RC, Marcondes JB, Chimelli LM, Fontes R, Niemeyer P et al. Quantitative analysis of somatostatin receptor subtypes (1-5) gene expression levels in somatotropinomas and correlation to in vivo hormonal and tumor volume responses to treatment with octreotide LAR. European Journal of Endocrinology 2008158 295-303. (doi:10.1530/ EJE-07-0562)

4 Salenave S, Boyce AM, Collins MT \& Chanson P. Acromegaly and McCune-Albright syndrome. Journal of Clinical Endocrinology and Metabolism 201499 1955-1969. (doi:10.1210/jc.2013.3826)

5 Stratakis CA, Kirschner LS \& Carney JA. Clinical and molecular features of the Carney complex: diagnostic criteria and recommendations for patient evaluation. Journal of Clinical Endocrinology and Metabolism 2001 86 4041-4046. (doi:10.1210/jcem.86.9.7903)

6 Kaltsas GA, Kola B, Borboli N, Morris DG, Gueorguiev M, Swords FM, Czirjak S, Kirschner LS, Stratakis CA, Korbonits M et al. Sequence analysis of the PRKAR1A gene in sporadic somatotroph and other pituitary tumours. Clinical Endocrinology 200257 443-448. (doi:10.1046/j.1365-2265.2002.01643.x)

7 Sandrini F, Kirschner LS, Bei T, Farmakidis C, Yasufuku-Takano J, Takano K, Prezant TR, Marx SJ, Farrell WE, Clayton RN et al. PRKAR1A, one of the Carney complex genes, and its locus (17q22-24) are rarely altered in pituitary tumours outside the Carney complex. Journal of Medical Genetics 200239 e78. (doi:10.1136/jmg.39.12.e78)

8 Yamasaki H, Mizusawa N, Nagahiro S, Yamada S, Sano T, Itakura M \& Yoshimoto K. GH-secreting pituitary adenomas infrequently contain inactivating mutations of PRKAR1A and LOH of 17q23-24. Clinical Endocrinology 200358 464-470. (doi:10.1046/j.1365-2265. 2003.01740.x)

9 Beuschlein F, Fassnacht M, Assie G, Calebiro D, Stratakis CA, Osswald A, Ronchi CL, Wieland T, Sbiera S, Faucz FR et al. Constitutive activation of PKA catalytic subunit in adrenal Cushing's syndrome. New England Journal of Medicine 2014370 1019-1028. (doi:10.1056/NEJMoa1310359)

10 Cao Y, He M, Gao Z, Peng Y, Li Y, Li L, Zhou W, Li X, Zhong X, Lei Y et al. Activating hotspot L205R mutation in PRKACA and adrenal Cushing's syndrome. Science 2014344 913-917. (doi:10.1126/science. 1249480)

11 Sato Y, Maekawa S, Ishii R, Sanada M, Morikawa T, Shiraishi Y, Yoshida K, Nagata Y, Sato-Otsubo A, Yoshizato T et al. Recurrent somatic mutations underlie corticotropin-independent Cushing's syndrome. Science 2014344 917-920. (doi:10.1126/science.1252328)

12 Forlino A, Vetro A, Garavelli L, Ciccone R, London E, Stratakis CA \& Zuffardi O. PRKACB and Carney complex. New England Journal of Medicine 2014370 1065-1067. (doi:10.1056/NEJMc1309730)
13 Larkin S, Reddy R, Karavitaki N, Cudlip S, Wass J \& Ansorge O. Granulation pattern, but not GSP or GHR mutation, is associated with clinical characteristics in somatostatin-naive patients with somatotroph adenomas. European Journal of Endocrinology 2013168 491-499. (doi:10.1530/EJE-12-0864)

14 Adams EF, Brockmeier S, Friedmann E, Roth M, Buchfelder M \& Fahlbusch R. Clinical and biochemical characteristics of acromegalic patients harboring gsp-positive and gsp-negative pituitary tumors. Neurosurgery 199333 198-203 (discussion 203). (doi:10.1227/ 00006123-199308000-00003)

15 Fougner SL, Casar-Borota O, Heck A, Berg JP \& Bollerslev J. Adenoma granulation pattern correlates with clinical variables and effect of somatostatin analogue treatment in a large series of patients with acromegaly. Clinical Endocrinology 201276 96-102. (doi:10.1111/ j.1365-2265.2011.04163.x)

16 Kola B, Korbonits M, Diaz-Cano S, Kaltsas G, Morris DG, Jordan S, Metherell L, Powell M, Czirjak S, Arnaldi G et al. Reduced expression of the growth hormone and type 1 insulin-like growth factor receptors in human somatotroph tumours and an analysis of possible mutations of the growth hormone receptor. Clinical Endocrinology 200359 328-338. (doi:10.1046/j.1365-2265.2003.01851.x)

17 Lyons J, Landis CA, Harsh G, Vallar L, Grunewald K, Feichtinger H, Duh QY, Clark OH, Kawasaki E, Bourne HR et al. Two G protein oncogenes in human endocrine tumors. Science 1990249 655-659. (doi:10.1126/science.2116665)

18 Spada A, Arosio M, Bochicchio D, Bazzoni N, Vallar L, Bassetti M \& Faglia G. Clinical, biochemical, and morphological correlates in patients bearing growth hormone-secreting pituitary tumors with or without constitutively active adenylyl cyclase. Journal of Clinical Endocrinology and Metabolism 199071 1421-1426. (doi:10.1210/ jcem-71-6-1421)

19 Yang I, Park S, Ryu M, Woo J, Kim S, Kim J, Kim Y \& Choi Y. Characteristics of gsp-positive growth hormone-secreting pituitary tumors in Korean acromegalic patients. European Journal of Endocrinology 1996134 720-726. (doi:10.1530/eje.0.1340720)

20 Bhayana S, Booth GL, Asa SL, Kovacs K \& Ezzat S. The implication of somatotroph adenoma phenotype to somatostatin analog responsiveness in acromegaly. Journal of Clinical Endocrinology and Metabolism 200590 6290-6295. (doi:10.1210/jc.2005-0998)

21 Mazal PR, Czech T, Sedivy R, Aichholzer M, Wanschitz J, Klupp N \& Budka H. Prognostic relevance of intracytoplasmic cytokeratin pattern, hormone expression profile, and cell proliferation in pituitary adenomas of akromegalic patients. Clinical Neuropathology 200120 163-171.

22 Obari A, Sano T, Ohyama K, Kudo E, Qian ZR, Yoneda A, Rayhan N, Mustafizur Rahman M \& Yamada S. Clinicopathological features of growth hormone-producing pituitary adenomas: difference among various types defined by cytokeratin distribution pattern including a transitional form. Endocrine Pathology 200819 82-91. (doi:10.1007/ s12022-008-9029-z)

Received 1 July 2014

Revised 11 September 2014

Accepted 15 September 2014 